\title{
Cardioplegia sangüínea anterógrada intermitente normotérmica e hipotérmica: estudo comparativo em corações agudamente isquêmicos de coelhos
}

\author{
Alfredo José RODRIGUES*, Walter Villela Andrade VICENTE*, João José CARNEIRO*, \\ Solange BASSETTO*
}

RBCCV 44205-508

Rodrigues A J, Vicente W V A, Carneiro J J, Bassetto S - Cardioplegia sangüínea anterógrada intermitente normotérmica e hipotérmica: estudo comparativo em corações agudamente isquêmicos de coelhos. Rev Bras Cir Cardiovasc 2000; 15 (3): 238-50.

RESUMO: Objetivo: Comparar, em corações agudamente isquêmicos de coelhos, estudados in vitro, o efeito bioquímico e inotrópico da infusão anterógrada intermitente de solução cardioplégica sangüínea hipercalêmica normo ou hipotérmica.

Material e Métodos: Foram analisadas as concentrações de glicogênio, lactato e a respiração mitocondrial e a Dp/dt $\mathrm{max}_{\text {ax }}$ do ventrículo esquerdo. As cardioplegias foram infundidas a cada 20 minutos, durante 60 minutos. O estudo compreendeu as Fase I e II, cada uma envolvendo 4 grupos: Controle, sem isquemia prévia; Isquemia, submetido a estudo bioquímico após 20 minutos de isquemia normotérmica; Normotermia (cardioplegia a $37^{\circ} \mathrm{C}$ ) e Hipotermia (cardioplegia a $17^{\circ} \mathrm{C}$ ). Na Fase I, sem reperfusão, procedeu-se apenas o estudo bioquímico. Na Fase II, com reperfusão, fez-se o estudo funcional (dP/dt) e o metabólico ao final de reperfusão.

Resultados: Ao final de 20 minutos de isquemia normotérmica houve queda significativa dos níveis de glicogênio, do consumo de $\mathrm{O}_{2}$ durante o estado 3 da respiração mitocondrial, da razão do controle respiratório (RCR) e elevação nos níveis de lactato, em relação ao controle. A infusão de cardioplegia hipotérmica elevou os níveis de glicogênio e restabeleceu os parâmetros da respiração mitocondrial, mas não alterou o lactato. A cardioplegia normotérmica não alterou os níveis de glicogênio e lactato, obtidos após a isquemia aguda, mas provocou queda maior no consumo de $\mathrm{O}_{2}$ e na RCR. Na Fase II, os valores bioquímicos retornaram a valores semelhantes aos do Controle, com ambas as cardioplegias. A dP/dt max $_{\text {ax }}$ do VE não foi significativamente diferente entre os grupos Normo e Hipotermia.

Conclusão: Ambas as soluções cardioplégicas resultaram em alterações bioquímicas similares ao final da reperfusão e foram igualmente eficazes em preservar a função do VE de coração de coelhos submetidos a período prévio de 20 minutos de isquemia global, em normotermia.

DESCRITORES: Parada cardíaca induzida, métodos. Miocárdio, metabolismo. Coração, fisiologia. Isquemia miocárdica, fisiopatologia. Função ventricular esquerda, fisiologia.

Trabalho realizado na Faculdade de Medicina de Ribeirão Preto da Universidade de São Paulo (FMRP-USP). Ribeirão Preto, SP, Brasil. Recebido para publicação em fevereiro de 2000.

* Da Divisão de Cirurgia Torácica e Cardiovascular da Faculdade de Medicina de Ribeirão Preto.

Endereço para correspondência: Alfredo José Rodrigues. Divisão de Cirurgia Torácica e Cardiovascular do Hospital das Clínicas da Faculdade de Medicina de Ribeirão Preto - USP, Hospital das Clínicas, Campus Universitário. Ribeirão Preto, SP, Brasil. CEP:14048-900. Telefax: (16) 602-2497. e.mail:alfredo@ fmrp.usp.br 
Rodrigues A J, Vicente W V A, Carneiro J J, Bassetto S - Cardioplegia sangüínea anterógrada intermitente normotérmica e hipotérmica: estudo comparativo em corações agudamente isquêmicos de coelhos. Rev Bras Cir Cardiovasc 2000; 15 (3): 238-50.

\section{INTRODUÇÃO}

Desde sua introdução na prática clínica por LICHTENSTEIN et al. (1), em 1991, a utilização de cardioplegia sangüínea normotérmica em infusão contínua para proteção miocárdica ganhou o interesse de diversos pesquisadores (2-15). Todavia, controvérsias perduram acerca do tempo seguro de interrupções na infusão contínua, muitas vezes, necessárias durante as operações, ou entre as infusões intermitentes de soluções cardioplégicas sangüíneas em normotermia.

Embora a maioria dos trabalhos utilizassem intervalos de até 15 minutos (2-15), constatamos, em estudo prévio $(16,17)$, que a infusão intermitente de solução cardioplégica sangüínea normotérmica, a intervalos de 20 minutos, preserva a função mitocondrial do miocárdio ventricular, bem como a função ventricular esquerda. Apesar destes achados propiciarem a utilização da infusão intermitente de solução cardioplégica sangüínea normotérmica a intervalos de 20 minutos, deve-se ressaltar que essa investigação incluiu, apenas, corações normais, com reservas energéticas plenas e sem alterações metabólicas prévias, o que, certamente, contribuiu para o miocárdio suportar os intervalos isquêmicos entre as doses de cardioplegia.

Como corações alterados, sobretudo os isquêmicos e os com sobrecargas de pressão, provavelmente estejam menos aptos a suportar os períodos de isquemia normotérmica de 20 minutos, consideramos importante realizar outra investigação experimental em corações submetidos à condição adversa.

Desta forma, o presente trabalho foi desenvolvido com o objetivo de comparar, em corações agudamente isquêmicos de coelhos, estudados in vitro, os efeitos bioquímico e funcional da infusão anterógrada intermitente, a cada 20 minutos, de solução cardioplégica sangüínea hipercalêmica administrada normo ou hipotérmica.

\section{MATERIAL E MÉTODOS}

\section{Fases do Estudo}

A pesquisa constou de 2 fases. A primeira, denominada Fase I, incluiu 4 grupos e constou, apenas, de estudo bioquímico do miocárdio ventricular. A segunda, Fase II, incluiu, além da investigação bioquímica, análise da função contrátil do ventrículo esquerdo, e também estudou 4 grupos.

A Figura 1 resume o protocolo experimental.

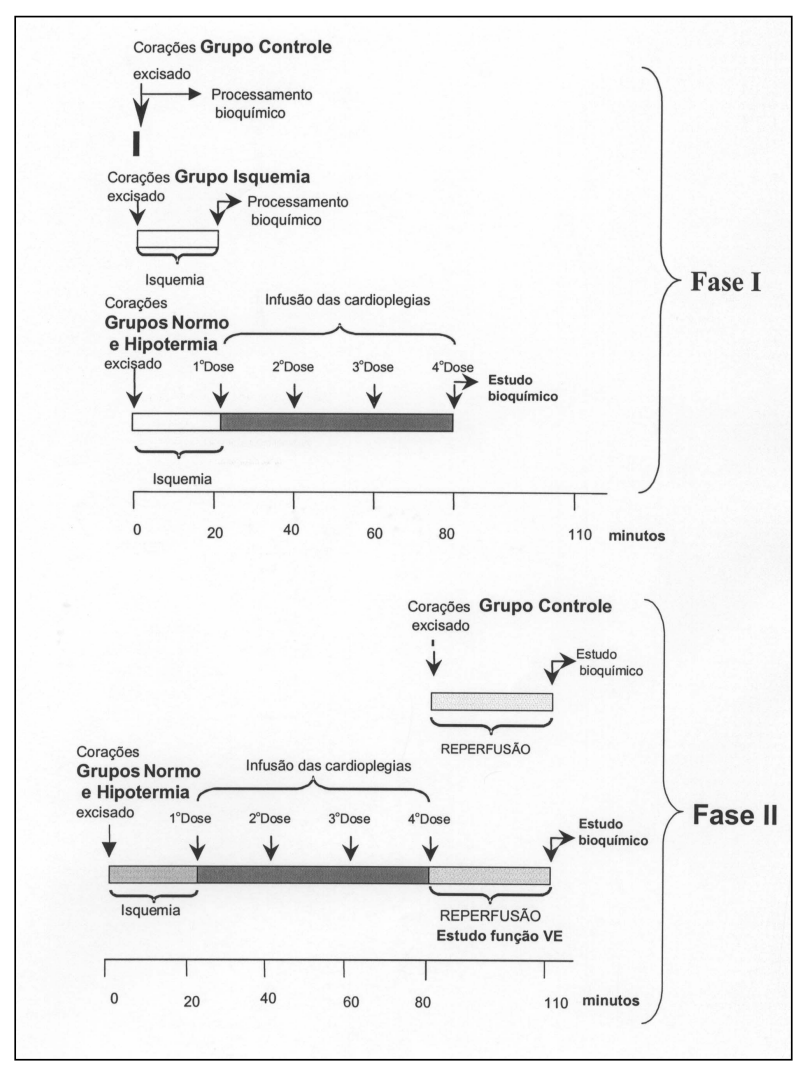

Fig. 1 - Representação esquemática do protocolo experimental, evidenciando-se os passos da investigação.

\section{Fase I: Estudo Metabólico}

Nesta fase analisaram-se, no miocárdio ventricular, tanto as concentrações de glicogênio e lactato, como a respiração mitocondrial nos grupos: Controle, Isquemia, Normotermia e Hipotermia.

- Grupo Controle - Este grupo foi constituído por corações $(n=18)$ enviados para estudo bioquímico imediatamente após cardiectomia.

- Grupo Isquemia - Constituído por corações $(n=15)$ que, após excisados, eram imersos em solução salina a $37^{\circ} \mathrm{C}$ por 20 minutos, sendo então, encaminhados para estudo bioquímico. Este grupo propiciou o estudo das alterações metabólicas advindas tão somente do período de 20 minutos de isquemia global em normotermia, servindo como referência para ambas as fases do estudo.

- Grupo Normotermia - Corações $(n=8)$ que, após receberem o mesmo tratamento do grupo Isquemia, eram submetidos à infusão anterógrada intermitente, a cada 20 minutos, de solução cardioplégica sangüínea a $37^{\circ} \mathrm{C}$, enquanto permaneciam em Beckers contendo solução salina a 0,9\%, 
Rodrigues A J, Vicente W V A, Carneiro J J, Bassetto S - Cardioplegia sangüínea anterógrada intermitente normotérmica e hipotérmica: estudo comparativo em corações agudamente isquêmicos de coelhos. Rev Bras Cir Cardiovasc 2000; 15 (3): 238-50.

também a $37^{\circ} \mathrm{C}$. Finda a última dose de cardioplegia, os corações eram encaminhados para estudo bioquímico.

- Grupo Hipotermia - Corações $(n=8)$ que receberam tratamento diferente do grupo Normotermia apenas quanto à temperatura da solução cardioplégica e do banho em que permaneciam imersos, ambos mantidos entre $15^{\circ} \mathrm{C}$ e $17^{\circ} \mathrm{C}$.

\section{Fase II: Estudo Metabólico e Funcional}

Incluiu um período de reperfusão, a fim de permitir a análise da função contrátil do ventrículo esquerdo subseqüente à administração das soluções cardioplégicas.

- Grupo Controle - Constituiu-se de corações $(n=7)$ que, imediatamente após excisados, foram perfundidos antes de serem encaminhados para a análise bioquímica, idêntica a realizada na Fase I.

- Grupo Isquemia - Este grupo foi o mesmo utilizado na fase I e não sofreu reperfusão. Todavia, os valores das variáveis bioquímicas deste grupo foram incluídos na análise estatística da Fase II visando a comparação com os resultados obtidos nos demais grupos após a reperfusão.

- Grupo Normotermia - Corações $(n=7)$ que receberam o mesmo tratamento do grupo Normotermia da Fase I. Entretanto, após o período de infusão da cardioplegia normotérmica, foram reperfundidos antes de serem encaminhados para a análise bioquímica.

- Grupo Hipotermia - Corações ( $n=7)$ submetidos a protocolo idêntico ao do grupo Normotermia, salvo pela temperatura da solução cardioplégica e do banho em que permaneceram imersos, ambos entre $15^{\circ} \mathrm{C}$ e $17^{\circ} \mathrm{C}$.

\section{Animais}

Usaram-se coelhos machos da raça Nova Zelândia, de 2 a $3 \mathrm{~kg}$ de peso. O procedimento anestésico e a cardiectomia foram realizados conforme descrito anteriormente (16).

\section{Cardioplegia}

Todas as soluções cardioplégicas foram feitas utilizando-se solução de Ringer à qual se adicionava sangue arterial na proporção de $40 \%$ do volume total.

O sangue arterial era colhido da carótida dos animais que seriam submetidos a cardiectomia (16 $\mathrm{ml}$ ), e da artéria carótida de outro coelho, cujo coração era, posteriormente, retirado para controle. Este animal recebia, imediatamente após cada sangria, transfusão de igual volume de sangue coletado na cavidade torácica do coelho que acabara de ser sacrificado pela cardiectomia e sua pressão arterial mantida a $70 \mathrm{mmHg}$, mediante infusão de alíquotas de solução salina, caso necessário. Em momento algum foram utilizadas drogas para suporte cardiovascular.

À primeira dose de cardioplegia, de $40 \mathrm{ml}$, seguiram-se as demais, de $20 \mathrm{ml}$ cada, todas sob pressão constante, de $100 \mathrm{~cm}$ de água.

\section{Reperfusão}

Utilizou-se um "Reservatório de Solução Cardioplégica" (Macchi Engenharia Biomédica-Ltda, São Paulo) e um "Módulo de Cardioplegia" (modelo CAP 30, Macchi Engenharia Biomédica-Ltda, São Paulo).

A reperfusão, com duração de 30 minutos, foi realizada mediante a infusão de solução de Krebs $\left(\mathrm{KCl}-11,5 \mathrm{~g} / \mathrm{l}, \mathrm{KH}_{2} \mathrm{PO}_{4}-21,0 \mathrm{~g} / \mathrm{l}, \mathrm{MgSO}_{4}-18,8 \mathrm{~g} /\right.$ I, $\mathrm{CaCl}_{2}-12,2 \mathrm{~g} / \mathrm{l}, \mathrm{NaHCO}_{3}-39,0 \mathrm{~g} / \mathrm{l}, \mathrm{NaCl}-6,75$ g/l, D-glicose- $2,0 \mathrm{~g} / \mathrm{l}$ ) a $37^{\circ} \mathrm{C}$, aerada com mistura de $95 \%$ de $\mathrm{O}_{2}$ e $5 \%$ de $\mathrm{CO}_{2}$, e infundida a pressão de $60 \mathrm{mmHg}$.

Os corações do grupo Controle, Normotermia e Hipotermia foram reperfundidos simultaneamente e todos receberam estimulação artificial a 180 b.p.m. com marcapasso epicárdico (modelo 5880 D, Medtronic).

\section{Obtenção da Pressão Intraventricular Esquerda e Cálculo da $d P / d t_{\text {max }}$}

A pressão intraventricular foi obtida por meio de um cateter-balão introduzido na cavidade ventricular esquerda, através do átrio esquerdo, e transdutor de pressão (Strain Gauge Coupler modelo 7179 Narco Bio Systems Inc., Houston, Texas, EEUU), com sensibilidade de $8 \mathrm{microvolts} / \mathrm{cm}$. Ao se fazerem os registros das pressões intraventriculares, o balão era distendido até atingir-se pressão diastólica de $25 \mathrm{mmHg}$.

As curvas de pressão foram registradas num polígrafo de inscrição mecânica de 4 canais ("Physiograph MK-IV", Narco Bio Systems Inc., Houston, Texas, EEUU).

O cálculo da $\mathrm{dP} / \mathrm{dt}_{\max }$ do ventrículo esquerdo foi realizado manualmente a partir dos traçados obtidos aos 10, 15 e 30 minutos após o inicio da reperfusão (18). 
Rodrigues A J, Vicente W V A, Carneiro J J, Bassetto S - Cardioplegia sangüínea anterógrada intermitente normotérmica e hipotérmica: estudo comparativo em corações agudamente isquêmicos de coelhos. Rev Bras Cir Cardiovasc 2000; 15 (3): 238-50.

\section{Estudo Bioquímico}

Em todos os corações o processamento bioquímico iniciava-se pela excisão dos ventrículos ao nível do sulco atrioventricular, seguida da imersão em solução salina a $0,9 \%$, a $4^{\circ} \mathrm{C}$, contidas em Beckers envoltos em gelo picado.

\section{Dosagem do Glicogênio Miocárdico}

A extração do glicogênio foi feita a partir de 500 $\mathrm{mg}$ de músculo cardíaco conforme o método de SJOGREEN et al. (19) e a dosagem de glicogênio, segundo HASSID \& ABRAHAN (20).

\section{Respiração Mitocondrial}

Obteve-se a fração mitocondrial pela técnica de SORDAHL et al. (21).

O consumo mitocondrial de oxigênio foi determinado polarograficamente a $30 \circ \mathrm{C}$, mediante a utilização de oxígrafo (modelo Gilson 5/6 Oxygraph, da Gilson Medical Eletronics, Inc., W. Beltline Middeton, EEUU).

Os parâmetros da fosforilação oxidativa foram calculados de acordo com CHANCE \& WILLIAMS (22) e ESTABROOK \& PULLMAN (23).

A concentração de proteína no homogeneizado foi determinada posteriormente, pelo método de LOWRY et al. (24).

\section{Dosagem do Lactato Miocárdico}

Foram realizadas conforme proposto por JENNINGS et al. (25) empregando-se espectrofotometria (Beckmann modelo DU 640B, EEUU), com comprimento de onda de $340 \mathrm{~nm}$, conforme descrito por GUTMANN \& WAHLEFELD (26).

\section{Análise Estatística}

A análise estatística foi realizada com o programa GraphPad Prism 2, versão 2.01, (GraphPad Software, Inc., San Diego, CA , EEUU). As comparações entre os grupos foram realizadas mediante 0 teste de Kruskal-Wallis, para a comparações entre mais de 2 grupos. Quando se evidenciou diferença significativa com o teste de KruskalWallis, realizou-se o teste de Dunn, para múltiplas comparações, 2 a 2 , entre os grupos, a fim de verificar onde ocorria a diferença. Em todos os testes, consideraram-se significativas as diferenças com valores de $p<0,05$.

\section{RESULTADOS}

Os resultados, demonstrados nas Tabelas $1 \mathrm{a}$ 5 expressam a média \pm desvio padrão e a mediana.

O menor tamanho das amostras ( $n$ ) demonstradas nas tabelas, quando confrontados com aqueles descritos nos grupos experimentais, são devido ao fato de que os valores bioquímicos e/ou do estudo funcional de alguns corações foram descartados devido a problemas técnicos evidenciados durante a realização dos mesmos.

\section{Glicogênio}

\section{Os resultados encontram-se na Tabela 1}

$\mathrm{Na}$ fase I, houve redução significativa dos níveis de glicogênio após 20 minutos de isquemia (grupo Isquemia), em relação ao Controle. Ao final do período de infusão intermitente de solução cardioplégica, os valores observados no grupo Normotermia eram semelhantes àqueles do grupo Isquemia, e significativamente menores em relação ao Controle. No grupo Hipotermia, embora a concentração se mantivesse baixa ao final da infusão cardioplégica, a diferença observada, em relação ao Controle, não foi significativa, todavia, não se observou diferença entre os grupos Normotermia e Hipotermia.

$\mathrm{Na}$ fase II, após reperfusão, os níveis de glicogênio no grupo Normotermia mantiveram-se significativamente abaixo dos observados no grupo Controle. Embora no grupo Hipotermia os níveis de glicogênio estivessem menores do que os do grupo controle, a diferença não foi significativa. Também não foi significativa a diferença entre os grupos Normotermia e Hipotermia.

Os níveis de glicogênio do grupo Isquemia foram significativamente menores em comparação aos observados no Controle dessa fase.

\section{Lactato}

Os valores estão demonstrados na Tabela 2 .

$\mathrm{Na}$ fase I, observou-se aumento significativo dos níveis de lactato do miocárdio ventricular após 20 minutos de isquemia a $37^{\circ} \mathrm{C}$ (grupo Isquemia), em relação ao Controle. Embora no final do período de infusão intermitente de solução cardioplégica a concentração de lactato, nos grupos Normo e Hipotermia, fosse inferior à observada após 20 minutos de isquemia normotérmica (grupo Isquemia), permaneceu significativamente maior que a concentração observada no grupo Controle, não havendo diferença significativa entre grupos Normotermia e Hipotermia a esse respeito. 
Rodrigues A J, Vicente W V A, Carneiro J J, Bassetto S - Cardioplegia sangüínea anterógrada intermitente normotérmica e hipotérmica: estudo comparativo em corações agudamente isquêmicos de coelhos. Rev Bras Cir Cardiovasc 2000; 15 (3): 238-50.

\section{TABELA 1}

\begin{tabular}{|c|c|c|c|c|}
\hline & CONTROLE & ISQUEMIA & NORMOTERMIA & HIPOTERMIA \\
\hline FASE I & $\begin{array}{c}0,34 \pm 0.16 \\
0,31 \\
(n=18)\end{array}$ & $\begin{array}{c}0,14 \pm 0,01^{*} \\
0,10 \\
(n=15) \\
p<0,01\end{array}$ & $\begin{array}{c}0,12 \pm 0,08 \text { * } \\
0,10 \\
(n=8) \\
p<0,01\end{array}$ & $\begin{array}{c}0,18 \pm 0,08 \\
0,20 \\
(n=8)\end{array}$ \\
\hline FASE II & $\begin{array}{c}0,28 \pm 0,08 \\
0,27 \\
(n=6)\end{array}$ & $\begin{array}{c}0,14 \pm 0,01 * * \\
0,10 \\
(n=15) \\
p<0,05\end{array}$ & $\begin{array}{c}0,11 \pm 0,03 * * \\
0,09 \\
(n=5) \\
p<0,05\end{array}$ & $\begin{array}{c}0,14 \pm 0,02 \\
0,13 \\
(n=6)\end{array}$ \\
\hline
\end{tabular}

Estão expressos os valores de p quando ocorreu diferença estatisticamente significativa. Os valores assinalados com * são estatisticamente diferentes do controle da Fase I e aqueles com ** são estatisticamente diferentes do controle da Fase II. O número de corações estudados em cada grupo encontra-se entre parêntesis.

TABELA 2

CONCENTRAÇÃO DE LACTATO NO MIOCÁRDIO VENTRICULAR, MÉDIA \pm DESVIO PADRÃO E MEDIANA, EXPRESSA EM $\mathrm{mmol} / \mathrm{ml}$

\begin{tabular}{|c|c|c|c|c|}
\hline & CONTROLE & ISQUEMIA & NORMOTERMIA & HIPOTERMIA \\
\hline FASE I & $\begin{array}{c}2,76 \pm 1,0 \\
2,7 \\
(n=18)\end{array}$ & $\begin{array}{c}11,05 \pm 3,5^{*} \\
11,0 \\
(n=15) \\
p<0,001\end{array}$ & $\begin{array}{c}8,80 \pm 3,3^{*} \\
9,0 \\
(n=8) \\
p<0,01\end{array}$ & $\begin{array}{c}9,12 \pm 2,3^{*} \\
9,0 \\
(n=8) \\
p<0,01\end{array}$ \\
\hline FASE II & $\begin{array}{c}1,93 \pm 1,0 \\
1,6 \\
(n=6)\end{array}$ & $\begin{array}{c}11,05 \pm 3,5 \text { ** } \\
11,0 \\
(n=15) \\
p<0,001\end{array}$ & $\begin{array}{c}3,10 \pm 1,0 " \\
2,8 \\
(n=5) \\
p<0,01\end{array}$ & $\begin{array}{c}3,10 \pm 1,0 " \\
3,5 \\
(n=6) \\
p<0,05\end{array}$ \\
\hline
\end{tabular}

Estão expressos os valores de "p" quando ocorreu diferença estatisticamente significativa. Os valores assinalados com “*” são estatisticamente diferentes do Controle da Fase I, aqueles assinalados com "**" são diferentes do Controle da Fase II e com " " aqueles diferentes em relação ao Isquêmico.

Na Fase II, após a reperfusão, a concentração de lactato reduziu-se acentuadamente, atingindo valores semelhantes ao observado no Controle e significativamente menor que a presente após o período de isquemia normotérmica (grupo Isquemia).

\section{Respiração Mitocondrial}

\section{Fase I}

Os valores encontram-se na Tabela 3.

No estado 3 , a isquemia de 20 minutos de duração a $37^{\circ} \mathrm{C}$ (grupo Isquemia) causou queda significativa no consumo de oxigênio mitocondrial, em relação ao grupo Controle.

Após a infusão intermitente da solução cardioplégica normotérmica a respiração mitocondrial manteve-se significativamente menor que a do grupo Controle, em contraste com os valores observados no grupo Hipotermia, que não diferiram do grupo Controle.

Vinte minutos de isquemia a $37^{\circ} \mathrm{C}$ (grupo Isquemia) não afetaram significativamente a razão do controle respiratório (RCR), embora ao final do período de infusão da solução normotérmica houvesse redução significativa da RCR em relação ao grupo Controle.

Não se observaram alterações significativas no comportamento das demais variáveis da respiração mitocondrial, nos 4 grupos.

\section{Fase II}

Os valores estão demonstrados na Tabela 4 . Após a reperfusão, as variáveis de respiração 
Rodrigues A J, Vicente W V A, Carneiro J J, Bassetto S - Cardioplegia sangüínea anterógrada intermitente normotérmica e hipotérmica: estudo comparativo em corações agudamente isquêmicos de coelhos. Rev Bras Cir Cardiovasc 2000; 15 (3): 238-50.

TABELA 3

VARIÁVEIS DA RESPIRAÇÃO MITOCONDRIAL NO MIOCÁRDIO VENTRICULAR, MÉDIA \pm DESVIO PADRÃO E MEDIANA, NA FASE I. OS VALORES DO CONSUMO DE OXIGÊNIO NO ESTADOS 3 E 4 ESTÃO EM NANOÁTOMOS DE OXIGÊNIO/MG DE PROTEÍNA/MIN

\begin{tabular}{|c|c|c|c|c|}
\hline & $\underset{(n=7)}{\text { CONTROLE }}$ & $\begin{array}{c}\text { ISQUÊMIA } \\
(n=8)\end{array}$ & $\begin{array}{c}\text { NORMOTERMIA } \\
(n=6)\end{array}$ & $\begin{array}{l}\text { HIPOTERMIA } \\
(\mathrm{n}=6)\end{array}$ \\
\hline Estado III & $\begin{array}{c}260,80 \pm 42,24 \\
272,3\end{array}$ & $\begin{array}{c}166,40 \pm 32,70^{*} \\
160,1 \\
p<0,05\end{array}$ & $\begin{array}{c}146,00 \pm 34,48 \text { * } \\
149,0 \\
p<0,01\end{array}$ & $\begin{array}{c}242,30 \pm 70,94 \text { ** } \\
226,3 \\
p<0,05\end{array}$ \\
\hline Estado IV & $\begin{array}{c}28,95 \pm 9,48 \\
27,76\end{array}$ & $\begin{array}{c}21,84 \pm 6,5 \\
22,5\end{array}$ & $\begin{array}{c}30,36 \pm 12,29 \\
27,6\end{array}$ & $\begin{array}{c}27,60 \pm 9,24 \\
25,78\end{array}$ \\
\hline RCR & $\begin{array}{c}9,50 \pm 2,48 \\
9,3\end{array}$ & $\begin{array}{c}8,11 \pm 2,24 \\
8,6\end{array}$ & $\begin{array}{c}5,13 \pm 1,85 \text { ** } \\
5,9 \\
p<0,05\end{array}$ & $\begin{array}{c}9,13 \pm 2,17 \\
9,7\end{array}$ \\
\hline ADP:O & $\begin{array}{c}2,36 \pm 0,45 \\
2,4\end{array}$ & $\begin{array}{c}2,65 \pm 0,77 \\
2,8\end{array}$ & $\begin{array}{c}1,98 \pm 0,55 \\
1,85\end{array}$ & $\begin{array}{c}2,53 \pm 0,30 \\
2,5\end{array}$ \\
\hline
\end{tabular}

Estão expressos os valores de "p" quando ocorreu diferença estatisticamente significativa. Assinalaram-se com "*” os valores estatisticamente diferentes do Controle e com "**” aqueles diferentes do grupo Normotermia.

TABELA 4

VARIÁVEIS DA RESPIRAÇÃO MITOCONDRIAL NO MIOCÁRDIO VENTRICULAR, MÉDIA \pm DESVIO PADRÃO E MEDIANA, DOS CORAÇÕES DA FASE II. OS VALORES DO CONSUMO DE OXIGÊNIO NO ESTADO 3 E 4 ESTÃO EM NANOÁTOMOS DE OXIGÊNIO/MG DE PROTEÍNA/MIN

\begin{tabular}{|c|c|c|c|c|}
\hline & $\begin{array}{c}\text { CONTROLE } \\
(n=7)\end{array}$ & $\underset{\substack{\text { ISQUEMIA } \\
(n=8)}}{ }$ & $\begin{array}{l}\text { NORMOTERMIA } \\
(\mathbf{n}=7)\end{array}$ & $\begin{array}{l}\text { HIPOTERMIA } \\
(n=7)\end{array}$ \\
\hline Estado III & $\begin{array}{c}145,40 \pm 36,95 \\
134,3\end{array}$ & $\begin{array}{c}166,40 \pm 32,70 \\
160,0\end{array}$ & $\begin{array}{c}113,90 \pm 36,20 \\
125,1\end{array}$ & $\begin{array}{c}128,90 \pm 43,65 \\
138\end{array}$ \\
\hline Estado IV & $\begin{array}{c}17,81 \pm 4,17 \\
16,6\end{array}$ & $\begin{array}{c}21,84 \pm 6,5 \\
22,5\end{array}$ & $\begin{array}{c}17,74 \pm 3,85 \\
18,4\end{array}$ & $\begin{array}{c}21,48 \pm 5,12 \\
21,16\end{array}$ \\
\hline $\mathrm{RCR}$ & $\begin{array}{c}8,43 \pm 2,33 \\
7,9\end{array}$ & $\begin{array}{c}8,11 \pm 2,24 \\
8,6\end{array}$ & $\begin{array}{c}6,70 \pm 2,62 \\
5,3\end{array}$ & $\begin{array}{c}6,31 \pm 2,41 \\
5,5\end{array}$ \\
\hline ADP:O & $\begin{array}{c}2,76 \pm 0,60 \\
2,8\end{array}$ & $\begin{array}{c}2,65 \pm 0,77 \\
2,8\end{array}$ & $\begin{array}{c}3.05 \pm 0,75 \\
3,2\end{array}$ & $\begin{array}{c}2,84 \pm 0,59 \\
2,9\end{array}$ \\
\hline
\end{tabular}

Não ocorreram diferenças significativas entre os grupos.

mitocondrial mostraram-se semelhantes às observadas no grupo Controle, sem diferença significativa entre os grupos Normotermia, Hipotermia e Isquemia.

Também não houve diferença significativa entre os valores observados nos grupos Controle e Isquemia.

\section{Função Ventricular Esquerda ( $d P / d t_{\text {max }}$ )}

A Tabela 5 demonstra os resultados da $\mathrm{dP} /$ $\mathrm{dt}_{\max }$.
Não observaram-se diferenças significativas nos valores da $\mathrm{dP} / \mathrm{dt}$ max entre os grupos Controle, Normotermia e Hipotermia.

\section{COMENTÁRIOS}

$\mathrm{Na}$ presente investigação, em que a assistolia foi desencadeada pela isquemia normotérmica global, as reservas de glicogênio sofreram significativa queda em relação ao controle, findos os 20 minutos de isquemia normotérmica, ponto de partida para o início do período de infusão cardioplégica. Conse- 
Rodrigues A J, Vicente W V A, Carneiro J J, Bassetto S - Cardioplegia sangüínea anterógrada intermitente normotérmica e hipotérmica: estudo comparativo em corações agudamente isquêmicos de coelhos. Rev Bras Cir Cardiovasc 2000; 15 (3): 238-50.

TABELA 5

\begin{tabular}{cccc}
\hline \multicolumn{4}{c}{ VALORES DA $\mathrm{dP} / \mathrm{dt}_{\max }$ DO VENTRÍCULO ESQUERDO, MÉDIA \pm DESVIO PADRÃO E MEDIANA, EM mmHg/s } \\
\hline & $\begin{array}{c}\text { CONTROLE } \\
(\mathbf{n}=5)\end{array}$ & $\begin{array}{c}\text { NORMOTERMIA } \\
(\mathbf{n}=5)\end{array}$ & $\begin{array}{c}\text { HIPOTERMIA } \\
(\mathbf{n}=5)\end{array}$ \\
\hline $\mathrm{dP} / \mathrm{dt}_{\max }$ & $1.552 \pm 457,1$ & $1.596 \pm 521,5$ & $1.240 \pm 697,6$ \\
$(\mathrm{mmHg})$ & 1.257 & 1.900 & 1.428 \\
\hline
\end{tabular}

Não ocorreram diferenças significativas entre os grupos.

qüentemente, ambos os grupos partiram de uma situação idêntica, ou seja, com baixa concentração de glicogênio e alta concentração de lactato miocárdico, decorrentes de 20 minutos de isquemia em normotermia. Ao término das infusões cardioplégicas, embora a concentração de glicogênio no grupo Hipotermia tendesse a ser maior, a diferença em relação ao grupo Normotermia não era significativa, e eram semelhantes as concentrações de lactato. Isto nos demonstra que ambos evoluíram, do ponto de vista do metabolismo glicolítico, para uma situação final semelhante.

Todavia, nossos resultados são insuficientes para esclarecer se as taxas metabólicas, durante os períodos das infusões cardioplégicas, eram semeIhantes, a despeito da diferença de temperatura.

É possível que, de forma mais pronunciada com a cardioplegia normotérmica, as infusões propiciassem metabolismo aeróbio, promovendo oxidação da glicose, remoção do lactato e síntese de glicogênio, ao passo que nos intervalos das infusões a anaerobiose desencadeou a glicogenólise, com produção de lactato, propiciando balanço final favorável a que as concentrações de substratos resultassem semelhantes às existentes no início do período de infusão das soluções cardioplégicas. Essa hipótese baseia-se nos estudos que averiguaram as conseqüências metabólicas, para o miocárdio, advindas da isquemia e infusão intermitente de solução cardioplégica sangüínea $(11,27,28)$.

Nesses estudos, DESLAURIERS et al. (27) e TIAN et al. (28) analisaram, utilizando espectroscopia de ressonância magnética, os efeitos da infusão intermitente de cardioplegia sangüínea normotérmica. Observaram que embora houvesse redução nos níveis de fosfocreatina e no $\mathrm{pH}$ intracelular e elevação do fósforo inorgânico, entre as infusões, suas concentrações eram completamente restauradas ao fim de cada dose de cardioplegia e durante a reperfusão, sem que resultasse prejuízo da função ventricular esquerda. Todavia em 1997, OLIVEIRA et al. (11), utilizando a mesma metodologia, embora encontrassem resultados semelhantes aos de
DESLAURIERS et al. (27) e TIAN et al. (28), observaram que apenas nos corações submetidos a períodos de isquemia de até 15 minutos não houve deterioração funcional.

Outro efeito benéfico dessas infusões de solução cardioplégica foi o de remover outros produtos nocivos do metabolismo isquêmico, além do lactato, e diminuir a acidose tecidual, embora, segundo ROUSLIN (29).e LEMASTERS et al. (30), a elevação do $\mathrm{pH}$ intracelular, nessa situação, pode ser deletéria à célula.

Nossos resultados são insuficientes para detalhar o metabolismo miocárdico nas duas diferentes temperaturas nas quais as soluções cardioplégicas sangüíneas foram administradas, todavia, o comportamento da função ventricular esquerda permite concluir que o metabolismo foi eficaz em preservar a integridade contrátil. Se houve lesão tecidual, provavelmente pôde ser reparada durante a reperfusão ou não foi suficiente para causar repercussão ventricular esquerda inotrópica passível de detecção com a metodologia empregada.

Duas observações, embora não analisadas objetivamente, contribuem para concluir pela competência metabólica dos corações durante a infusões cardioplégicas não resultando em lesão miocárdica grave, a despeito das baixas reservas energéticas. Uma, a de que nenhum coração apresentou arritmia ventricular que inviabilizasse a análise da função ventricular esquerda, não obstante algumas extrasístoles, que cessavam em poucos minutos, indicando que o metabolismo glicolítico durante o período de infusão cardioplégica foi eficaz em preservar a função da membrana celular (31-35). KING \& OPIE (36), em recente revisão, lembram, ainda, que a produção de ATP mediante glicólise nas fases iniciais da reperfusão, parece ser essencial à prevenção do colapso energético e funcional. Deve-se considerar, ainda, a homeostasia do $\mathrm{Ca}^{++}$, mediante a preservação da atividade da $\mathrm{Na}^{+} / \mathrm{K}^{+}$ATPase ${ }^{(37,38)}$ da membrana citoplasmática. A outra, o fato de que alguns corações apesar de terem apresentado, à palpação, contratura do 
Rodrigues A J, Vicente W V A, Carneiro J J, Bassetto S - Cardioplegia sangüínea anterógrada intermitente normotérmica e hipotérmica: estudo comparativo em corações agudamente isquêmicos de coelhos. Rev Bras Cir Cardiovasc 2000; 15 (3): 238-50.

miocárdio ventricular ao final dos 60 minutos de infusão intermitente da solução cardioplégica, recuperarem plenamente a contratilidade durante a reperfusão. Todavia, há certa controvérsia se a contratura isquêmica é ou não um indicativo de lesão irreversível, embora contratura crescente continue considerada indício de progressão da lesão celular (36).

GOODWIN et al. (39), bem como, FRASER et al. (40) e HENNING et al. (41) demonstraram que a glicogenólise e a glicogênese ocorrem simultaneamente durante a perfusão em condições de aerobiose, em corações isolados de rato, com balanço positivo para a glicogênese. Tal balanço entre glicogênese e glicogenólise ocorre também durante períodos de isquemia grave por redução acentuada do fluxo coronário, predominando a glicogenólise. Durante a reperfusão a atividade glicolítica e de glicogênese ainda se encontram elevadas, mas em níveis semelhantes $(39,40)$ resultando em concentrações estáveis de glicogênio. Na impossibilidade de obter energia anaerobiamente a partir de ácidos graxos, ocorre redução acentuada na concentração de glicogênio, pois esse passa a ser o único substrato energético, a permitir que a glicogenólise sobrepuje a glicogênese $(40,41)$.

Acreditamos ser esse o motivo pelo qual as concentrações miocárdicas de glicogênio do grupo Controle da Fase II serem pouco menores que as da Fase I, pois foram dosadas ao final do período de reperfusão em que a glicose foi a única fonte de substrato. Explica-se assim, também, a permanência das baixas concentrações de glicogênio nos grupos Hipotermia e Normotermia.

O uso de proteínas recombinantes fotossensíveis ou indicadores fluorescentes, captados pelas mitocôndrias, permitiu demonstrar que essas organelas mostram grandes alterações nos níveis de $\mathrm{Ca}^{++}$na matriz após a abertura de poros ou canais do retículo endoplasmático ou da membrana celular, ensejando especulações acerca do possível papel da cinética do $\mathrm{Ca}^{++}$mitocondrial na homeostasia do cálcio intracelular, também em condições normais (42).

No Brasil, KREISEL (43), em 1996, mediante trabalho experimental utilizando corações de coeIhos submetidos à cardioplegia, mostrou liberação de $\mathrm{K}^{+}$mitocondrial durante a fase de "swelling" que não era reabsorvido quando da reversão do "swelling" mitocondrial. Esse aspecto foi confirmado, posteriormente, por ICHAS et al. (44), em trabalho no qual os autores apresentam as mitocôndrias como organelas excitáveis capazes de gerar e transmitir, além de sinais elétricos, sinais de cálcio que tanto podem contribuir na modulação dos níveis de $\mathrm{Ca}^{++}$ intracelular, como representarem um meio adicional pelo qual a excitabilidade mitocondrial influencia vários processos citosólicos, sugerindo que a mitocôndria tem outras funções importantes para a manutenção da homeostasia celular, além da fosforilação oxidativa.

No presente trabalho, optamos por metodologia que nos possibilitaria o estudo das mitocôndrias como geradoras de ATP em aerobiose e o estudo da via energética alternativa de produção de ATP em condições de anaerobiose, a via glicolítica.

A análise da função mitocondrial indica que 20 minutos de isquemia a $37^{\circ} \mathrm{C}$ resultaram em diminuição na capacidade mitocondrial de consumir oxigênio durante o estado 3 da respiração. Todavia, como desapareceram as diferenças na respiração mitocondrial, pode-se concluir que esse prejuízo funcional da organela não foi decorrente de lesão irreversível, e que embora a infusão intermitente de solução cardioplégica normotérmica não o levasse a se recuperar, como o fez a hipotérmica, tampouco induziu prejuízo adicional. Outros investigadores também observaram alterações na respiração mitocondrial, durante isquemia, similares às nossas $(45,46)$.

Vários autores investigaram um marcador bioquímico que se correlacione com as alterações funcionais e, ao mesmo tempo, seja preditivo de recuperação funcional após isquemia miocárdica. Alguns autores observaram correlação entre a capacidade oxidativa e a capacidade em recuperar a função mecânica ventricular (47-49), em contrapartida, outros não observaram tal correlação $(50,51)$. Talvez esses resultados divergentes possam ser atribuídos às diferentes metodologias empregadas pelos vários autores.

Devemos considerar, ainda, que a falta de correlação entre a capacidade oxidativa dos miócitos, sobretudo quando avaliada mediante o estudo da respiração mitocondrial, e a recuperação funcional mecânica, pode advir do fato de que as mitocôndrias provavelmente têm funções diversificadas (44), como salientamos anteriormente. Ademais, resultados preliminares sugerem que, além da diversidade funcional intrínseca, há diversidade funcional entre as mitocôndrias de tecidos diferentes de uma mesma espécie (Ródio, L.B.C*) e entre espécies (Sader, A.A. e Roselino, J.E.S.**).

É portanto evidente a controvérsia a respeito do melhor marcador bioquímico de lesão isquêmica, bem como de seu valor preditivo na recuperação funcional do miocárdio. Nesta investigação optou-se pelo estudo da respiração mitocondrial, como a proposta de ROSENKRANZ et al. (48), tanto por sua similaridade com as condições clínicas de utilização de soluções cardioplégicas, como pela experiência prévia de nosso laboratório com o método (16-17). 
Rodrigues A J, Vicente W V A, Carneiro J J, Bassetto S - Cardioplegia sangüínea anterógrada intermitente normotérmica e hipotérmica: estudo comparativo em corações agudamente isquêmicos de coelhos. Rev Bras Cir Cardiovasc 2000; 15 (3): 238-50.

ROSENKRANZ et al. (48) observaram redução persistente no consumo de $\mathrm{O}_{2}$ no estado 3 da respiração mitocondrial, mas não no estado 4 , nem no ritmo de fosforilação oxidativa do miocárdio de cães reperfundidos após 45 minutos de isquemia normotérmica. Resultados semelhantes foram observados por outros autores $(50,52)$.

Deve-se, porém, considerar que a técnica de obtenção da fração mitocondrial proposta por SORDHAL et al. (21), a qual utilizamos, é traumática (53), para a grande maioria das mitocôndrias. Dessa forma, os valores obtidos podem corresponder apenas à pequena porção de mitocôndrias que puderam suportar o processo de isolamento, geralmente as que se encontram em melhor estado, incorrendose no erro de subestimar os efeitos da isquemia sobre a função mitocondrial, como já advertira CLEMENTS et al. (54), em 1980, ao encontrarem resultados semelhantes com relação ao estado 3 e a RCR após isquemia miocárdica de 20 minutos.

De qualquer forma, optamos por um parâmetro adicional funcional, a dP/dtmax, que nos permitiria avaliar eventuais respostas cujo substrato bioquímico não fosse evidenciado pela metodologia empregada.

Não obstante a cardioplegia sangüínea normotérmica com infusão contínua ter sido empregada com sucesso, deve-se salientar que a proposição original de LICHTENSTEIN et al. (1) baseouse, apenas, em considerações teóricas, tendo gerado várias questões, ainda hoje, à espera de elucidação. Uma, que tem preocupado alguns investigadores, refere-se ao período de tempo durante o qual o miocárdio pode suportar as interrupções na infusão da cardioplegia contínua normotérmica, por vezes necessárias, como por exemplo, durante a confecção das anastomoses nas artérias coronárias, nas operações de revascularização do miocárdio. Outra, que consideramos pertinente, refere-se à possibilidade de que embora a infusão intermitente de solução cardioplégica sangüínea em normotermia, a cada 20 minutos, se mostrasse segura em corações normais de coelhos $(16,17)$, faltava-nos investigar o emprego do método em corações previamente isquêmicos, objetivo desta pesquisa.

Trabalhos experimentais têm demonstrado que a interrupção ou infusão intermitente de soluções cardioplégicas sangüíneas é prejudicial (55-58), embora recentemente, GOMES (58), avaliando pacientes portadores de estenose aórtica operados sob infusão intermitente de soluções cardioplégicas sangüíneas em normo ou hipotermia, concluiu que ambas as soluções não foram eficientes, do ponto de vista bioquímico, em proteger o miocárdio.

Apesar do potencial malefício inerente à infusão de soluções cardioplégicas sangüíneas normotérmicas ${ }^{(55-58)}$, mesmo por curtos períodos, essa tática ganhou adeptos na prática clínica que relataram resultados iguais ou superiores aos obtidos com soluções hipotérmicas $(3,6,8-10,12,15)$.

JACQUET et al. publicaram (7), em 1999, os resultados de estudo prospectivo aleatório em pacientes submetidos à revascularização do miocárdio, comparando a cardioplegia sangüínea normotérmica intermitente anterógrada, infundida a intervalos nunca superiores a 15 minutos, com a cristalóide hipotérmica, infundida por via anterógrada e retrógrada a cada 60 minutos ou quando necessário. Observaram que a morbidade e a mortalidade observadas em ambos os grupos não diferiram, embora a cardioplegia normotérmica provocasse menos lesão miocárdica, o que é evidenciado pelos menores níveis plasmáticos de CK-MB e Troponina I.

Contudo, nesse mesmo ano, ERICSSON et al. (5) publicaram os resultados de estudo experimental, em porcos, no qual compararam a infusão contínua de cardioplegia sangüínea normotérmica com a intermitente, infundida a cada 10 minutos, por um período de 45 minutos, sob circulação extracorpórea. Observaram que a infusão intermitente foi ineficaz em preservar a função global do ventrículo esquerdo, embora em ambos os grupos houvesse deterioração similar da função diastólica.

Pelos trabalhos citados, pode-se concluir que permanece controversa a segurança da interrupção da infusão contínua de solução cardioplégica sangüínea em normotermia. Embora a maioria dos estudos clínicos não demonstre danos evidentes, com intervalos isquêmicos em torno de 15 minutos. Todavia, a maioria das pesquisas envolveu, sobretudo, pacientes com boa reserva funcional miocárdica, o que pode tornar difícil a detecção de malefícios, a longo prazo, que poderiam contribuir para menor sobrevida dos pacientes. Certamente o principal motivo pelo qual os relatos clínicos e experimentais somam-se, sem dirimir a questão, está em apresentarem diferenças marcantes nos protocolos, concordantes apenas no que se refere à temperatura de infusão da solução cardioplégica em normotermia.

Nossos dados mostram que, embora a reperfusão tenha restabelecido ambos, a função mitocondrial e o metabolismo glicolítico aeróbio, e que não houve déficit funcional, o miocárdio em ambos os grupos, Normotermia e Hipotermia, manteve-se às custas do metabolismo anaeróbio nos intervalos entre as infusões cardioplégicas. É evidente que esses efeitos metabólicos são pouco desejáveis para uma metodologia cujo emprego, experimental ou clínico, visa proteger o miocárdio de novas lesões e, se possível, proporcionar a recuperação de lesões prévias. Ademais, a observa- 
Rodrigues A J, Vicente W V A, Carneiro J J, Bassetto S - Cardioplegia sangüínea anterógrada intermitente normotérmica e hipotérmica: estudo comparativo em corações agudamente isquêmicos de coelhos. Rev Bras Cir Cardiovasc 2000; 15 (3): 238-50.

ção de contratura em alguns corações, aliada às alterações bioquímicas verificadas na Fase I, parece indicar que os mesmos estiveram próximos à linha demarcatória entre lesão isquêmica reversível e irreversível.

Embora nossos resultados possam contraindicar a utilização, ou alertar para uso de cautela no emprego de cardioplegia normotérmica intermitente, com intervalos de 20 minutos, deve-se ressaltar que, por outro lado, a hipotermia também não ofereceu proteção miocárdica superior. Na verdade, embora as diferenças na $\mathrm{dP} / \mathrm{dt}_{\max }$ entre o grupo Normotermia e Hipotermia não fossem estatisticamente significativas, houve tendência a valores menores com a hipotermia.

É possível que o melhor caminho seja o do meio termo, caracterizado pelo uso de cardioplegia sangüínea tépida ou isotérmica, em relação à temperatura da sala operatória, possivelmente associada à indução normotérmica sugerida por ROSENKRANZ $(59,60)$ e à cardioplegia secundária normotérmica, sugerida por LAZAR et al. (61).

Outro fator a considerar na avaliação de nossos resultados é de que o período de isquemia normotérmica que precedeu o período de infusão da cardioplegia, pode, ainda, ter pré-condicionado os corações do grupo Normotermia, tornando-os mais aptos a suportar os intervalos de isquemia que se seguiram $(62,63)$, sobretudo se considerarmos que a hipotermia pode afetar negativamente o précondicionamento ${ }^{(64,65)}$, privando o grupo Hipotermia deste efeito. Todavia, o protocolo empregado não nos permite uma análise objetiva acerca da ocorrência de pré-condicionamento.
Três outras observações merecem ainda ser consideradas quanto à metodologia empregada nesta investigação. A primeira, de que os resultados obtidos neste estudo com a utilização de corações de coelhos podem não corresponder ao de outros animais, aí incluído o homem. Outro aspecto relevante diz respeito à ausência de sangue na solução de reperfusão, o que pode ter minimizado o aparecimento de lesões miocárdicas dela decorrentes, uma vez que se reconhece a participação de elementos figurados do sangue na patogênese das lesões de reperfusão ${ }^{(66)}$. Finalmente, deve-se considerar que os coelhos dos quais o coração foi retirado para compor o grupo Controle da reperfusão, Fase II, também colheu-se sangue para o preparo das soluções cardioplégicas, cerca de 90 minutos antes da retirada do órgão. Embora nesse período se mantivessem adequadamente anestesiados e hemodinamicamente estáveis, podem ter sofrido estresse metabólico que influenciou os resultados bioquímicos e funcionais, de modo a comprometer a comparação com os demais grupos, na fase II.

\section{CONCLUSÃO}

A infusão anterógrada intermitente, de soluções cardioplégicas sangüíneas a temperaturas de $37^{\circ} \mathrm{C}$ e entre 15 e $17^{\circ} \mathrm{C}$, a cada 20 minutos, provocou alterações bioquímicas similares e foi igualmente eficaz em preservar a função ventricular esquerda de coração de coelhos submetidos a período prévio de 20 minutos de isquemia global, em normotermia. 
Rodrigues A J, Vicente W V A, Carneiro J J, Bassetto S - Cardioplegia sangüínea anterógrada intermitente normotérmica e hipotérmica: estudo comparativo em corações agudamente isquêmicos de coelhos. Rev Bras Cir Cardiovasc 2000; 15 (3): 238-50.

RBCCV 44205-508

Rodrigues A J, Vicente W V A, Carneiro J J, Bassetto S - Normothermic and hypothermic intermittent anterograde blood cardioplegia: comparative study in acutely ischemic rabbit hearts. Rev Bras Cir Cardiovasc 2000; 15 (3): 238-50.

ABSTRACT: Objective: This in vitro investigation aimed to compare the degree of myocardial preservation afforded by intermittent anterograde normothermic $v s$ hypothermic blood cardioplegia, infused at 20 minutes intervals, in acutely ischemic rabbit hearts.

Methods: Myocardial concentration of glycogen and lactate, as well as mitochondrial respiration and left ventricular function ( $\mathrm{dP} / \mathrm{dtmax}$ ) were analyzed. The study was divided into two phases, one without (Phase I) and one with (Phase II) a period of reperfusion following the last dose of cardioplegia. Each phase included 4 groups. In Phase I, hearts sent for metabolic analysis immediately after being excised comprised the Control group. After being subjected to global normothermic ischemia for $20 \mathrm{~min}$, hearts were sent for biochemical analysis (Ischemic group), or else received 4 doses of cardioplegia at $37^{\circ} \mathrm{C}$ (Normothermic group) or at $17^{\circ}$ $\mathrm{C}$ (Hypothermic group) before biochemical analysis was performed. In Phase II, except for the Ischemic group, left ventricular function was assessed on a modified Langendorff apparatus preceding metabolic analysis.

Results: Global normothermic ischemia (Ischemic group) resulted in reduction of glycogen levels, $\mathrm{O}_{2}$ consumption during state 3 and respiratory control rate (RCR) of mitochondrial respiration, and in elevation of lactate levels. Without reperfusion (Phase I), a significant biochemical improvement was noticed after infusion of 4 doses of hypothermic cardioplegia (Hypothermic group) except for the lactate levels that remained higher than the Control group. After normothermic cardioplegia (Normothermic group) biochemical variables showed values similar to the Control group. Reperfusion (Phase II) was followed by restoration of all biochemical variables to baseline (Control group). LV function showed similar results between Control, Normothermic and Hypothermic groups.

Conclusions: The intermittent antegrade infusion of blood cardioplegia at $37^{\circ} \mathrm{C}$ and $17^{\circ} \mathrm{C}$, infused at 20 minutes intervals, produced similar biochemical and functional results in rabbit hearts submitted to a prior period (20 minutes) of acute normothermic ischemia.

DESCRIPTORS: Heart arrest induced, methods. Myocardium, metabolism. Heart, physiology. Myocardial ischemia, physiopathology. Ventricular function, left, physiology.

\section{REFERÊNCIAS BIBLIOGRÁFICAS}

1 Lichtenstein S V, Ashe K A, el Dalati H, Cusimano R J, Panos A, Slutsky A S - Warm heart surgery. J Thorac Cardiovasc Surg 1991; 101: 269-74.

2 Ali I M \& Kinley C E - The safety of intermittent warm blood cardioplegia. Eur J Cardiothorac Surg 1994; 8: 554-6.

3 Calafiore A M, Teodori G, Mezzetti A et al. - Intermittent antegrade warm blood cardioplegia. Ann Thorac Surg 1995; 59: 398-402.

4 Christakis G T, Koch J P, Deemar K A et al. - A randomized study of the systemic effects of warm heart surgery. Ann Thorac Surg 1992; 54: 449-59.

5 Ericsson A B, Kawakami T, Vaage J - Intermittent warm blood cardioplegia does not provide adequate myocardial resuscitation after global ischaemia. Eur J Cardiothoracic Surg 1999; 16: 233-9.

6 Isomura T, Hisatomi K, Sato T, Hayashida N, Ohishi K - Interrupted warm blood cardioplegia for coronary artery bypass grafting. Eur J Cardiothorac Surg 1995; 9: 133-8.

7 Jacquet $\mathrm{L} \mathrm{M}$, Noirhomme $\mathrm{P} \mathrm{H}$, Van Dyck $\mathrm{M} \mathrm{J}$ et al. Randomized trial of intermittent antegrade warm blood versus cold crystalloid cardioplegia. Ann Thorac Surg 1999; 67: 471-7.
8 Landymore R, Murphy J T, Hall R, Islam M - Randomized trial comparing intermittent antegrade warm blood cardioplegia with multidose cold blood cardioplegia for coronary artery bypass. Eur J Cardiothorac Surg 1996; 10: 179-84.

9 Lichtenstein S V, Naylor C D, Feindel C M et al. - Intermittent warm blood cardioplegia. Warm Heart Investigators. Circulation 1995; 92 (9 Suppl): II341-6.

10 Mezzetti A, Calafiore A M, Lapenna D et al. - Intermittent antegrade warm cardioplegia reduces oxidative stress and improves metabolism of the ischemic-reperfused human myocardium. J Thorac Cardiovasc Surg 1995; 109:787-95.

11 Oliveira N C, Boeve T J, Torchiana D F et al. - Ischemic intervals during warm blood cardioplegia in the canine heart evaluated by phosphorus 31-magnetic resonance spectroscopy. J Thorac Cardiovasc Surg 1997; 114: 1170-80.

12 Pelletier L C, Carrier M, Leclerc Y, Cartier R, Wesolowska $\mathrm{E}$, Solymoss B C - Intermittent antegrade warm versus cold blood cardioplegia: a prospective, randomized study. Ann Thorac Surg 1994; 58: 41-9.

13 Salerno T A, Houck J P, Barroso C A M et al. - Retrograde continuous warm blood cardioplegia: a new concept in myocardial protection. Ann Thorac Surg 1991; 51: 245-7. 
Rodrigues A J, Vicente W V A, Carneiro J J, Bassetto S - Cardioplegia sangüínea anterógrada intermitente normotérmica e hipotérmica: estudo comparativo em corações agudamente isquêmicos de coelhos. Rev Bras Cir Cardiovasc 2000; 15 (3): 238-50.

14 Vaughn C C, Opie J C, Florendo F T, Lowell P A, Austin J - Warm blood cardioplegia. Ann Thorac Surg 1993; 55: 1227-32.

15 Yau T M, Ikonomidis J S, Weisel R D et al. - Which techniques of cardioplegia prevent ischemia? Ann Thorac Surg 1993; 56: 1020-8.

16 Rodrigues A J, Sader A A, Vicente W V A, Bassetto S - Cardioplegia sangüínea normotérmica intermitente anterógrada: estudo experimental em coelhos. Rev Bras Cir Cardiovasc 1997; 12: 278-87.

17 Rodrigues A J, Sader A A, Vicente W V A, Bassetto S - Cardioplegia sangüínea normotérmica intermitente anterógrada. II. Com e sem aminoácidos: estudo comparativo em coelhos. Rev Bras Cir Cardiovasc 1997; 12: 340-7.

18 Aloan L - Análise da função ventricular. In: Aloan L, ed. Hemodinâmica e angiocardiografia. obtenção de dados, interpretação e aplicações clínicas. Rio de Janeiro: Atheneu, 1982: 123-80.

19 Sjogreen B, Nordenskjold T, Holmgren H, Mullerstromm $\mathrm{J}$ - Beitragzur kenntaris der leberthymithmic (glicogen, phosphos and calcium in der kaninchendeber). Arch F D Ges Physiol 1938; 240: 427.

20 Hassid W Z \& Abrahan S - Chemical procedures for analysis of polysaccharides. I. Determination of glycogen and starch. In: Collowick S P, Kaplam N O, eds. Methods in enzymology. New York: Academic Press, 1957; Publishers. 34-50.

21 Sordhal L A, Johnson C, Blailok Z R, Schuwartz A - The mitochondrion. Meth Pharmacol 1971; 1: 247.

22 Chance B \& Williams G R - The respiratory chain and oxidative phosphorylation. Adv Enzimol 1956; 17: 65-134.

23 Estabrook R W \& Pullman M E - Oxidation and phosphorylation. Methods in Enzymology 1967; New York: Academic Press, 167: 7-8.

24 Lowry $\mathrm{O} H$, Rosebrough N J, Farr A L, Randall R J Protein measurement with the folinphenol reagent. $J$ Biol Chem 1951; 193: 265.

25 Jennings R B, Reimer K A, Steenbergen Jr. C, Schaper J - Total ischemia III: effect of inhibition of anaerobic glycolysis. J Mol Cell Cardiol 1989; 21 (Suppl. I): 37-54.

Gutmann I \& Wahlefeld A W - Methods of enzymatic analysis. New York: Academic Press, 1974; 3: 1464.

27 Deslauriers R, Tian G, Kupriyanov V, Lareau S, Salerno $T$ A - Basic research on myocardial protection: a magnetic resonance approach. In: Warm heart surgery . Chicago: Mosby-Yearbook, 1996: cap. 4. p. 31-45.

28 Tian G, Xiang B, Butler K W R et al. - A 31P-nuclear magnetic resonance study of intermittent warm blood cardioplegia. J Thorac Cardiovasc Surg 1995; 109: 1155-63.
29 Rouslin W - Protonic inhibition of mitochondrial olygomycin-sensitive adenosine 5-triphosphatase in ischemic and autolyzing cardiac muscle: possible mechanism for the mitigation of ATP hydrolysis under nonenergizing conditions. J Biol Chem 1983; 258: 9657-61.

30 Lemasters J T, Nieminen A L, Qian T, Trost L C, Herman $B$ - The mitochondrial permeability transition in toxic, hypoxic and reperfusion injury. Mol Cell Biochem 1997; 174: 159-65.

31 Cross H R, Opie L H, Radda G K, Clarke K - Is a high glycogen content beneficial or detrimental to the ischemic rat heart? A controversy resolved. Circ Res 1996; 78: 482-91.

32 Opie L H - Cardiac metabolism: emergence, decline and resurgence. Part I. Cardiovasc Res 1992; 26: 721-33.

33 Opie L H - Fuels: aerobic and anaerobic metabolism. In: Opie L H, ed. Heart: physiology, from cell to circulation. Philadelphia, 1998. cap. 11, p. 295-342.

34 Weiss $\mathrm{J} \mathrm{N}$ \& Hiltbrand B - Functional compartmentation of glycolytic versus oxidative metabolism in isolated rabbit heart. J Clin Invest 1985; 75: 436-47.

35 Weiss J N \& Lamp S T - Glycolysis preferentially inhibits ATP-sensitive $\mathrm{K}+$ channels in isolated guinea pig cardiac myocytes. Science 1987; 238: 67-9.

36 King L M \& Opie L H - Glucose and glycogen utilization in myocardial ischemia-changes in metabolism and consequences for the myocyte. Mol Cell Biochem 1998; 180: 3-26.

37 Jeremy R W, Koretsune Y, Marban E, Becker L C Relation between glycolysis and calcium homeostasis in postischemic myocardium. Circ Res 1992; 70: 1180-90.

38 Jeremy R W, Ambrosio G, Pike M M, Jacobus W E, Becker L C - The functional recovery of post-ischemic myocardium requires glycolysis during early reperfusion. J Mol Cell Cardiol 1993; 25: 261-76.

39 Goodwin G W, Arteaga J R, Taegtmeyer H - Glycogen turnover in the isolated working rat heart. $\mathrm{J} \mathrm{Biol} \mathrm{Chem}$ 1995; 270: 9234-40.

40 Fraser H, Lopaschuk G D, Clanachan A S - Assessment of glycogen turnover in aerobic, ischemic, and reperfused working rat hearts. Am J Physiol 1998; 275 (5 Pt 2): H1533-41.

41 Henning S L, Wambolt R B, Schonekess B O, Lopaschuk $G \mathrm{D}$, Allard M F - Contribution of glycogen to aerobic myocardial glucose utilization. Circulation 1996; 93: 1549-55.

42 Rutter G A, Fasolato C, Rizzuto R - Calcium and organelles: two-sided story. Biochem Biophys Res Commun 1998; 253: 549-57. 
Rodrigues A J, Vicente W V A, Carneiro J J, Bassetto S - Cardioplegia sangüínea anterógrada intermitente normotérmica e hipotérmica: estudo comparativo em corações agudamente isquêmicos de coelhos. Rev Bras Cir Cardiovasc 2000; 15 (3): 238-50.

43 Kreisel U - Avaliação comparativa de mitocôndrias de miocárdio de coelhos submetidos à cardioplegia básica. [Tese. Doutorado] Ribeirão Preto: Faculdade de Medicina de Ribeirão Preto, Universidade de São Paulo, 1996. 93p.

44 Ichas F, Jouaville L S, Mazat J P - Mitochondria are excitable organelles capable of generating and conveying electrical and calcium signals. Cell 1997; 89: 1145-53.

45 Flameng W, Andres J, Ferdinande P, Mattheussen M, Van Belle $\mathrm{H}$ - Mitochondrial function in myocardial stunning. J Mol Cell Cardiol 1991; 23: 1-11.

46 Lesnefsky E J, Tandler B, Ye J, Slabe T J, Turkaly J, Hoppel C L - Myocardial ischemia decreases oxidative phosphorylation through cytochrome oxidase in subsarcolemmal mitochondria. Am J Physiol 1997; 273 (3 Pt 2): H1544-54.

47 Zimmer S D, Ugurbil K, Michurski S P et al. - Alterations in oxidative function and respiratory regulation in the post-ischemic myocardium. J Biol Chem 1980; 264: 12402-11.

48 Rosenkranz E R, Okamoto F, Buckberg G D et al. Studies of controlled reperfusion after ischaemia II. Biochemical studies: failure of tissue adenosine triphosphate levels to predict recovery of contractile function after controlled reperfusion. $J$ Thorac Cardiovasc Surg 1986; 92(3 Pt 2): 488-501.

49 Marshall R C - Correlation of contractile dysfunction with oxidative energy production and tissue high energy phosphate stores during partial coronary flow disruption in rabbit heart. J Clin Invest 1988; 82: 86-95.

50 Edoute $\mathrm{Y}$, van der Merwe E, Sanan D, Kotzé J C, Steinmann C, Lochner A - Normothermic ischemic cardiac arrest of the isolated working rat heart: effects of time and reperfusion on myocardial ultrastructure, mitochondrial oxidative function and mechanical recovery. Circ Res 1983; 53: 663-78.

51 Saks V A, Kapelko V I, Kupriyanov V V et al. - Quantitative evaluation of relationship between cardiac energy metabolism and post-ischemic recovery of contractile function. J Mol Cell Cardiol 1989; 21 (Suppl. 1): 6778.

52 Wood J M, Hanley H G, Entman M L et al. - Biochemical and morphological correlates of acute experimental myocardial ischemia in the dog. IV- Energy mechanisms during very early ischemia. Circ Res 1979; 44: 52-61.

53 Shlafer M, Kirsh M, Lucchesi B R, Slater A D, Warren $S$ - Mitochondrial function after global cardiac ischemia and reperfusion: influence of organelle isolation protocols. Basic Res Cardiol 1981; 76: 250-66.

54 Clements I P, Dewey J D, Harrison Jr. C E - Failure of interventions to maintain mitochondrial function in ischemic myocardium. Mayo Clin Proc 1980; 55: $637-44$.

55 Matsuura H, Lazar H L, Yang X M, Rivers S, Treanor P $\mathrm{R}$, Shemin R J - Detrimental effects of interrupting warm blood cardioplegia during coronary revascularization. J Thorac Cardiovasc Surg 1993; 106: 357-61.

56 Mlsare B D, Krukenkamp I B, Caldarone C A, Levitsky $\mathrm{S}$ - Can continuous warm cardioplegia be interrupted? Surg Forum 1992; 43: 208-10.

57 Ko W, Zelano J, Fahey A L, Berman K, Isom O W, Krieger $\mathrm{K} \mathrm{H}$ - Ischemic tolerance of the arrested heart during warm cardioplegia. Eur J Cardiothorac Surg 1993; 7: 295-9.

58 Gomes W A - Efeitos das cardioplegias sangüíneas normotérmicas e hipotérmicas nos substratos intracelulares em pacientes submetidos a troca de valva aórtica. [Tese. Livre Docência] São Paulo: Escola Paulista de Medicina da Universidade Federal de São Paulo,1998. 73p.

59 Rosenkranz E R, Buckberg G D, Laks H, Mulder D G Warm induction of cardioplegia with glutamateenriched blood in coronary patients with cardiogenic shock who are dependent on inotropic drugs and intra aortic balloon support. J Thorac Cardiovasc Surg 1983; 86: 507-18.

60 Rosenkranz E R, Vinten-Johansen J, Buckberg G D, Okamoto F, Edwards H, Bugyi H - Benefits of normothermic induction of blood cardioplegia in energy-depleted hearts, with maintenance of arrest by multidose cold blood cardioplegic infusions. J Thorac Cardiovasc Surg 1982; 84: 667-77.

61 Lazar H L, Buckberg G D, Manganaro A J et al. - Reversal of ischemic damage with secondary blood cardioplegia. J Thorac Cardiovasc Surg 1979; 78: 688-97.

62 Hawaleshka A \& Jacobsohn E - Ischaemic preconditioning: mechanisms and potential clinical applications. Can J Anaesth 1998; 45: 670-82.

63 Meldrum D R - Mechanisms of cardiac preconditioning: ten years after the discovery of ischemic preconditioning. J Surg Res 1997; 73: 1-13.

64 Dote K, Wolff R A, Van Winkle D M - Hypothermia increases the threshold for ischemic preconditioning. J Thorac Cardiovasc Surg 1998; 116: 319-26.

65 Lu E X, Ying G L, Guo X - Hypothermia during preconditioned ischemia-reperfusion attenuates the myocardial protection of preconditioning. J Thorac Cardiovasc Surg 1997; 114: 514-6.

66 Lucchesi B R, Werns S W, Fantone J C - The role of the neutrophil and free radicals in ischemic myocardial injury. J Mol Cell Cardiol 1989; 21: 1241-51. 